\title{
Prognostic assessment of breast carcinoma submitted to neoadjuvant chemotherapy with pathological non-complete response
}

\author{
Uanderson Resende ${ }^{*}$ D, César Cabello, Susana Oliveira Botelho Ramalho and Luiz Carlos Zeferino
}

\begin{abstract}
Background: Breast cancer with pathological non-complete response (non-pCR) after neoadjuvant chemotherapy (NAC) has a worse prognosis. Despite Neo-Bioscore has been validated as an independent prognostic model for breast cancer submitted to NAC, non-pCR carcinoma was not assessed in this setting.

Methods: This is a retrospective trial that included women with localized breast cancer who underwent NAC and had non-pCR carcinoma in surgical specimen between 01/01/2013 to 12/31/2015 with a three-year follow-up. Survival analysis was performed by Kaplan-Meier estimator and hazard ratio (HR) set by log-rank test for the primary and secondary endpoints, respectively Disease-Free Survival (DFS) and Overall Survival (OS). According to Neo-Bioscore, the proposed prognostic model named Clustered Neo-Bioscore was classified into low (0-3), low-intermediate (4-5), high-intermediate (6) and high (7) risk. The prognostic accuracy for recurrence risk was assessed by time-dependent receiver operating characteristic (time-ROC) methodology. Multivariate Cox regression assessed the menopausal status, histological grade, Ki-67, estrogen receptor, HER2, tumor subtype, pathological and clinical stages. Confidence interval at 95\% (C195\%) and statistical significance at set 2-sided p-value less than 0.05 were adopted.

Results: Among the 310 women enrolled, 267 patients (86.2\%) had non-pCR carcinoma presenting size T3/T4 (63.3\%), node-positive axilla (74.9\%), stage III (62.9\%), Ki-67 $\geq 20 \%$ (71.9\%) and non-luminal A (78.3\%). Non-pCR carcinoma presented worse DFS-3y $(H R=3.88, C 195 \%=1.18-11.95)$ but not OS-3y $(H R=2.73, C 195 \%=0.66-11.40)$. Clustered NeoBioscore discerned the recurrence risk for non-pCR carcinoma: low (DFS-3y $=0.86$; baseline), low-intermediate (DFS$3 y=0.70 ; H R=2.61$ ), high-intermediate (DFS-3y=0.13, HR= 14.05), and high (DFS-3y = not achieved; HR= 22.19). The prognostic accuracy was similar between Clustered Neo-Bioscore and Neo-Bioscore (0.76 vs 0.78, $p>0.05)$. Triplenegative subtype $(H R=3.6, C \mid 95 \%=1.19-10.92)$ and pathological stages $\|(H R=5.35, C 195 \%=1.19-24.01)$ and III $(H R=6.56, C \mid 95 \%=1.29-33.32)$ were prognoses for low-intermediate risk, whereas pathological stage III $(H R=13.0, C \mid 95 \%=1.60-106.10)$ was prognosis for low risk.
\end{abstract}

Conclusions: Clustered Neo-Bioscore represents a novel prognostic model of non-pCR carcinoma undergoing NAC with a more simplified and appropriate score pattern in the assessment of prognostic factors.

Keywords: Breast, Carcinoma, Neoadjuvant therapy, Prognosis, Disease-free survival

\footnotetext{
*Correspondence: uandersonmed@gmail.com

Division of Gynecological and Mammary Oncology, Woman's Hospital Dr

José Aristodemo Pinotti (CAISM) of State University of Campinas (UNICAMP),

Rua Alexander Fleming 101, Campinas, São Paulo 13083-083, Brazil
}

(c) The Author(s). 2019 Open Access This article is distributed under the terms of the Creative Commons Attribution 4.0 International License (http://creativecommons.org/licenses/by/4.0/), which permits unrestricted use, distribution, and reproduction in any medium, provided you give appropriate credit to the original author(s) and the source, provide a link to the Creative Commons license, and indicate if changes were made. The Creative Commons Public Domain Dedication waiver (http://creativecommons.org/publicdomain/zero/1.0/) applies to the data made available in this article, unless otherwise stated. 


\section{Background}

Breast cancer is the most common malignant tumor in women worldwide with about $22 \%$ of all new cancers in 2015 [1]. In recent years, new data on the molecular heterogeneity of breast carcinoma have led to a greater understanding of the diversity of prognosis [2]. Neoadjuvant chemotherapy (NAC) is one of the main therapeutic modalities in the treatment of high-risk breast cancer [3] due to tumor downstaging with increased rate of conservative breast surgery [4], better model of clinical research to evaluate the performance of biomarkers and tumor sensitivity to chemotherapeutic agents [5], generally using a smaller sample size and shorter follow-up time [6].

The main prognostic factors of breast cancer are age, clinical performance, menopausal status, tumor stage [7], histological type, tumor differentiation, gene expression signature $[8,9]$, molecular subtype [10, $11]$, expression of estrogen (ER) $[12,13]$ and progesterone receptors (PR) [14], HER2 oncoprotein (HER2) [15] and cellular proliferation index Ki67 (Ki-67) [1618]. However, these factors alone have limited prognostic power while combining them into an algorithm increases the prognostic power [19]. Neo-Bioscore is currently the leading prognostic model for assessing the recurrence risk in women with breast cancer undergoing NAC. It is calculated from the sum of the scores obtained from the degree of tumor differentiation, ER, HER2, clinical and pathological stages [2022]. However, Neo-Bioscore has eight recurrence risk scores (0 to 7 ) whose survival curves presented a non-linear time-to-event distribution among them due to the non-uniform distance among time-to-event occurrences [21, 22].

Breast carcinoma undergoing NAC that did not present a pathological complete response (non-pCR) has a worse prognosis [23-27] and there is little information on its independent prognostic factors [28]. Therefore, it would be appropriate to assess a novel prognostic model that is more simplified to deal with linear stratification of scores for women with non-pCR carcinoma. We propose a novel prognostic model named Clustered Neo-Bioscore from the clustering of similar prognostic scores of Neo-Bioscore which is composed of four scores - low, lowintermediate, high-intermediate and high-risk whose risk strata are linearly more distanced from each other as to the time-to-event of tumor recurrence in the survival curve. Clustered Neo-Bioscore and Neo-Bioscore were compared for prognostic accuracy. Independent prognostic factors for non-pCR carcinoma were analyzed by Cox regression according to Neo-Bioscore, low and low-intermediate Clustered Neo-Bioscore.

\section{Methods}

\section{Patients}

This is a retrospective cohort that included women with localized breast carcinoma who underwent NAC followed by surgery at the Woman's Hospital Dr. José Aristodemo Pinotti (CAISM) of the State University of Campinas (UNICAMP), between 01/01/2013 to 12/31/ 2015. It was approved by the Ethics Committee in Research of the Faculty of Medical Sciences (FCM) of UNICAMP. A total of 345 women were selected but 35 patients were excluded due to neoadjuvant hormonal therapy (25), surgical contraindication (2), clinical comorbidities (2), or metastases diagnosed throughout NAC (6). Therefore, 310 women were enrolled in this analysis.

\section{Materials}

The clinical and biological factors analyzed were menopausal status, tumor size, axilla status, clinical stage, histologic grade, ER, Ki67, HER2 and molecular subtypes according to immunohistochemical findings [18, 29-31]. Pathologic analyzes and immunohistochemical assays of core needle biopsy and surgical specimen were performed in the same institutional laboratory. Menopausal status was defined as the presence of amenorrhea greater than 1 year or serum estradiol levels below $10 \mathrm{pg} / \mathrm{ml}$. The ER expression was classified by Allred scoring into negative, low (2-6) and high (7-8) [32]. The Ki-67 expression was subdivided into low $(<20 \%)$, intermediate $(20 \% \leq \mathrm{Ki} 67<50 \%)$ and high $(\geq 50 \%)$ [33, 34]. Molecular subtypes were luminal A, luminal B, HER2 and triplenegative (TN) based on the results of the immunohistochemistry assay [9, 29-32].

\section{NAC protocol}

Enrolled women received 4 cycles of doxorubicin $60 \mathrm{mg} /$ $\mathrm{m}^{2}$ and cyclophosphamide $600 \mathrm{mg} / \mathrm{m}^{2}$ at intervals of 3 weeks followed by 4 cycles of paclitaxel $210 \mathrm{mg} / \mathrm{m}^{2}$ at intervals 3 weeks $(4 \mathrm{AC}+4 \mathrm{~T})$ or 12 cycles of paclitaxel 80 $\mathrm{mg} / \mathrm{m}^{2}$ weekly $(4 \mathrm{AC}+12 \mathrm{~T})$ [35]. About $85 \%$ (63/74) of women with HER2 carcinoma also received trastuzumab at $4 \mathrm{mg} / \mathrm{kg}$ (initial dose) followed by $2 \mathrm{mg} / \mathrm{kg}$ weekly for 23 consecutive weeks plus 9 cycles at $6 \mathrm{mg} / \mathrm{kg}$ at intervals $3^{\circ}$ weeks [36]. Approximately 1\% (9/94) of women with $\mathrm{TN}$ carcinoma also received carboplatin $\mathrm{AUC}=1.5$ weekly associated with paclitaxel [37]. None of the women showed serious adverse effects that required discontinuation of NAC. No one dropped out of NAC due to loss of follow-up or weak treatment compliance. There were dose reductions and/or delays for 52 women by neuropathic (37) and emetic (9) toxicity, surgery anticipation (4) or no clinical response (2). Dose reduction by only $20 \%$ (43) and/or one cycle (4) was the majority and therefore without influencing the overall efficacy of 
NAC. The period between the last cycle of chemotherapy and surgery was 20-30 days. Surgical clips were used to identify the tumor location by mammography in the surgery. The clinical staging by TNM classification was performed by clinical examination, mammography, breast and axillary ultrasound, bone scan computed tomography of chest and abdominal $[38,39]$. Supportive care such as antiemetic, blood transfusion and antimicrobial therapy were given according to oncologist's discretion. The $\mathrm{pCR}$ was defined as the absence of invasive carcinoma in surgical breast specimen and micro or macrometastases in removed axillary lymph nodes [24, 27, 36-38].

\section{Treatment assessment}

The principal outcome was Disease-Free Survival (DFS) that was defined from the date of diagnosis until the date of death, locoregional or distant recurrence. The secondary outcome was Overall Survival (OS) that was defined from the date of diagnosis until the date of death. The follow-up time was 37 months. The NeoBioscore was calculated from the sum of the scores of the following data: clinical stage (I/IIA: 0 point, IIB/IIIA: 1 point, IIIB/IIIC: 2 points), pathological stage (0/I: 0 point; IIIB: 1 point, IIIC: 2 points), ER (negative: 1 point; positive: 0 point), nuclear grade (I/II: 0 point; III: 1 point) and HER2 (negative: 1 point; positive: 0 point) [19-22]. The Clustered Neo-Bioscore was calculated from Neo-Bioscore by grouping prognostic scores similar into each other to obtain four very different prognostic categories: low $(0,1,2$ or 3$)$, low-intermediate ( 4 or $5)$, high-intermediate (6) and high (7). This configuration of categories presented greater homogeneity within them and heterogeneity among them leading to a greater time-to-event distance for tumor recurrence among the categories.

\section{Statistical analysis}

Statistical analysis was performed by SPSS 22.0 software (version 22; SPSS Inc). Categorical variables were analyzed with Pearson's chi-square test and Kendal's Tau for multinomial regression according to pathological response and Neo-Bioscore. The Kaplan-Meier estimator was used to obtain the time-to-event for recurrence tumor of pCR and non-pCR carcinomas according to Neo-Bioscore and Clustered Neo-Bioscore, survival curves for a 3-year follow-up and the hazard ratio (HR) between pCR and non-pCR carcinomas for DFS-3y and OS-3y. The log-rank test was used to compare the prognosis among the risk scores of the pathological stage (I, II and III), Neo-Bioscore (0 to 7) and Clustered NeoBioscore (low, low-intermediate, high-intermediate and high). The mean time to first relapse (mTFR) and the respective HR among the risk categories of Clustered NeoBioscore were calculated by Kaplan-Meier, while the statistical significance was defined by the log-rank test. The multivariate analysis by Cox proportional hazards regression was performed to define the independent prognostic factors of non-pCR carcinoma according to Neo-Bioscore, low and low-intermediate Clustered-NeoBioscore. The Cox regression for intermediate-high and high risk was not performed because of the limited number of patients in these groups [40, 41]. The prognostic accuracy for recurrence risk among the pathological stage, Neo-Bioscore and Clustered Neo-Bioscore was assessed from the area under the curve (AUC) by time-dependent receiver operating characteristic (timeROC) methodology. The confidence interval at $95 \%$ (C195\%) was adopted and statistical significance was at set 2 -sided $p$-value less than $0.05(p<0.05)$ [42].

\section{Results}

\section{Patient characteristics}

Women with high-risk breast cancer prevailed with size T3/T4 (64\%), stage III (62\%), positive axilla (74\%), Ki$67 \geq 20 \%$ (74\%) and non-Luminal A (81\%). The pCR carcinoma was about $13.8 \%$ (43/310) of the total with histological grade III (62.8\%), negative ER (74.4\%), Ki-67 $\geq$ 20\% (88.4\%), HER2 and TN (93\%) subtypes, T3/T4 size $(69.8 \%)$, N1 axilla (48.8\%) and stage III (58.1\%). Otherwise, the non-pCR carcinoma presented similar clinical characteristics to pCR carcinoma with size T3/T4 (63.3\%), axilla N1 (43.8\%) and stage III (62.9\%) but different biological factors with histological grade I/II (53.9\%), ER Allred 7-8 (49.4\%), Ki-67 < 50\% (71.5\%) and Luminal B (30.3\%) (Table 1).

Most of the carcinomas had Neo-Bioscore $\leq 5$ (91.0\%; $282 / 310)$ with histological grade I/II (56.7\%; 160/282), positive ER (61.0\%; 172/282), Ki-67<50\% (70.2\%; 198/ 282), negative HER2 (74.8\%; 211/282), non-TN subtype (72.3\%; 204/282), size T3/T4 (60.6\%; 171/282), nodepositive axilla (71.2\%; 201/282) and stage III (62.1\%; 175/ 282). Carcinomas classified as Neo-Bioscore $=0-3$ (48.4\%; $150 / 310)$ prevailed in premenopausal status (55.3; 83/150), histological grade I/II (76.7\%; 115/150), ER Allred 7-8 (56.0\%; 84/150), Ki-67<50\% (64.0\%; 114/150), negative HER2 (66.7\%; 100/150), non-TN subtype (80.7\%; 121/ 150), size T1/T2 (58.7\%; 88/150), node-positive axilla (59.3\%; 89/150) and stage I/II (60.7\%; 91/150). Carcinomas classified as Neo-Bioscore $=4-5(42.6 \% ; 132 / 310)$ prevailed in postmenopausal status (58.3\%; 77/132), histological grade III $(65.9 \%$; 87/132), negative ER (50.8\%; 67/ 132), Ki-67 $\geq 20 \% \quad(75.8 \% ; 100 / 132)$, negative HER2 (84.1\%; 111/132), non-luminal A subtype (80.3\%; 106/ 132), size T3/T4 (82.6\%; 109/132), positive axilla (84.8\%; $112 / 132)$ and stage III (80.3\%; 106/132). Otherwise, carcinomas classified as Neo-Bioscore $=6-7(9.0 \% ; 28 / 310)$ prevailed in postmenopausal status $(64.3 \% ; 18 / 28)$, histological grade III (100\%; 28/28), negative ER (75.0\%; 21/28), 


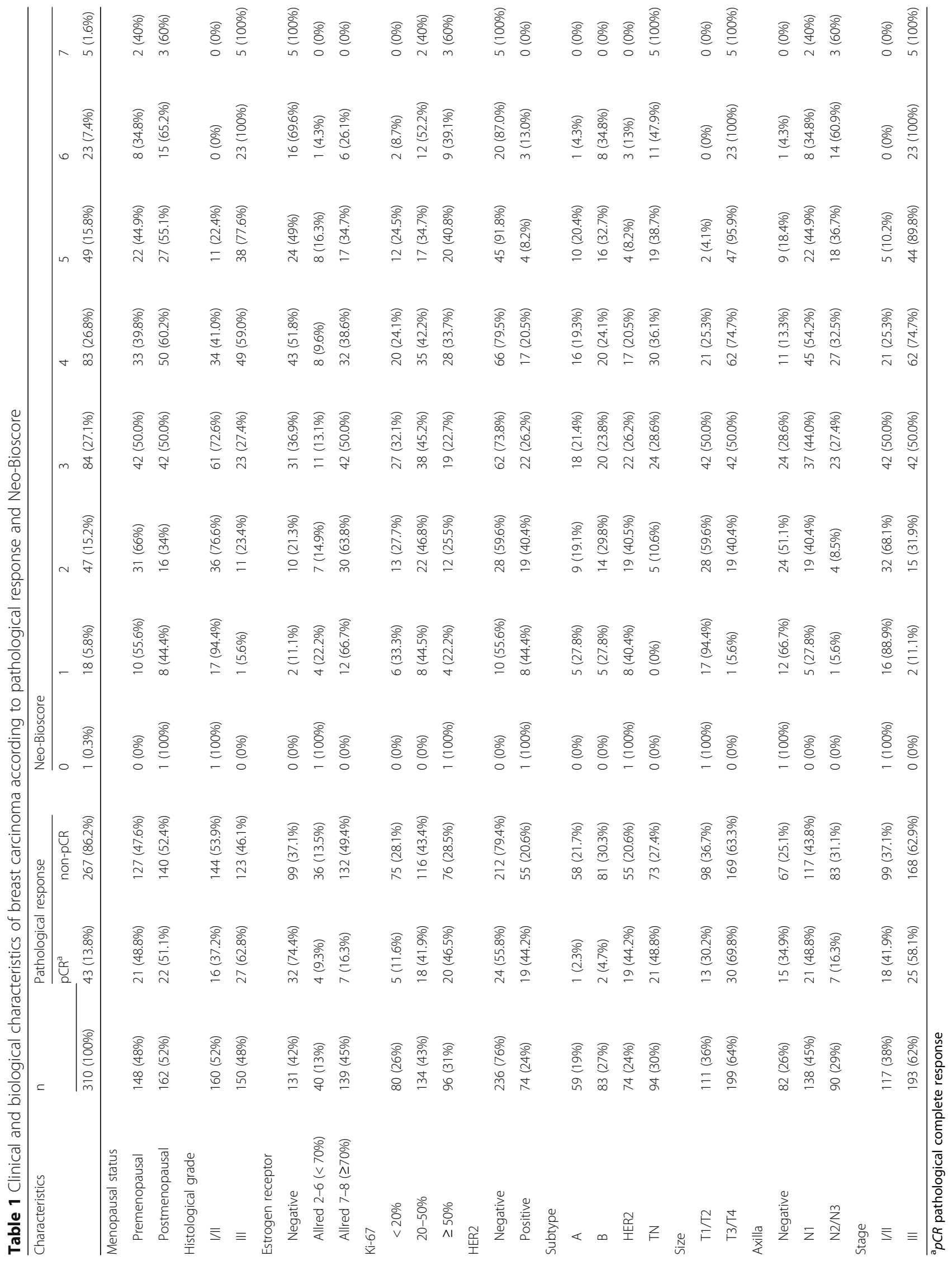


Ki-67 $\geq 20 \%$ (92.9\%; 26/28), negative HER2 (89.3\%; 25/28), TN subtype $(57.1 \% ; 16 / 28)$, size T3/T4 $(100 \% ; 28 / 28)$, positive axilla $(96.4 \% ; 27 / 28)$ and stage III $(100 \% ; 28 / 28)$ (Table 1).

\section{Survival analysis}

The women enrolled in this study presented median time from first recurrence (mTFR) equal to $1274 \mathrm{~d}$ (C195\%: 1219-1329), DFS-3y = 0.75 (CI95\%: 0.72-0.78), median overall survival (mOS) of 1405 d (CI95\%: 13601449) and OS-3y $=0.81$ (CI95\%: 0.79-0.84). The pCR carcinoma occurred in 43 women $(13.8 \%)$ with MTFR $=$ $1432 \mathrm{~d}$ (CI95\%: 1339-1525) and DFS-3y $=0.92$ (CI95\%: $0.88-0.96)$. Otherwise, 267 women had non-pCR carcinoma $(86.7 \%)$ with mTFR $=1244$ d $($ CI95\%: 1182-1305) and DFS-3y $=0.69$ (CI95\%: 0.65-0.73) (Table 2). At 3years non-pCR carcinoma showed a greater recurrence risk than pCR carcinoma with $\mathrm{HR}=3.75$ (CI95\%: 1.1811.95) but not the death risk with $\mathrm{HR}=2.73$ (CI95\%: 0.66-11.35) (Fig. 1).

Considering the group of 43 pCR carcinomas, $65.1 \%$ of the total $(28 / 43)$ was classified as Clustered Neo-Bioscore of low risk (Neo-Bioscore 0-3) with DFS-3y $=0.93$ (0.87$0.99)$ and $34.9 \%(15 / 43)$ was of low-intermediate risk (Neo-Bioscore 4-5) with DFS-3y $=0.92(0.86-0.98)$ (Table 2). For group of 267 non-pCR carcinomas, $45.7 \%$ (122/ 267) was of low risk with DFS-3y $=0.86$ (CI95\%: $0.82-$ $0.90), 43.8 \%(117 / 267)$ was of low-intermediate risk with DFS-3y $=0.70$ (CI95\%: 0.65-0.75), 8.6\% (23/267) was of high-intermediate risk (Neo-Bioscore 6) with DFS-
$3 y=0.13$ (CI95\%: 0.05-0.21) and 1.9\% (5/267) was of high risk (Neo-Bioscore 7$)$ with DFS-3y $=0 \quad(\mathrm{mTFR}=$ 475 d) (Table 2).

The subdivision of the scores for pathological staging (I, II and III), Neo-Bioscore (0 to 7) and Clustered Neo-Bioscore (low, low-intermediate, highintermediate and high) presented statistical significance in relation to the risk of tumor recurrence for non-pCR carcinoma $(p<0.01)$ (Fig. 2). The prognostic accuracy was assessed by the time-ROC model $(p>0.05)$ between Neo-Bioscore (0.78, CI95\%: $0.71-$ 0.84 ) and Clustered Neo-Bioscore (0.76; CI95\%: 0.69 0.83). Otherwise, these markers presented greater accuracy $(\mathrm{p}<0.01)$ than the pathological stage $(0.69$; C195\%: 0.63-0.76) (Fig. 3).

The Clustered Neo-Bioscore subgroups of non-pCR carcinoma had remarkably different recurrence risks with statistical significance among them $(\mathrm{p}<0.01)$. Considering the low risk as reference $(\mathrm{mTFR}=1388 \mathrm{~d})$, the low-intermediate risk presented $\mathrm{mTFR}=1247 \mathrm{~d}(\mathrm{HR}=$ 2.61, CI95\%: 1.33-5.11), the high-intermediate risk had $\mathrm{mTFR}=618 \mathrm{~d}(\mathrm{HR}=14.05, \mathrm{CI} 95 \%: 6.78-29.11)$ and the high risk showed $\mathrm{mTFR}=433 \mathrm{~d}(\mathrm{HR}=22.19, \mathrm{CI} 95 \%$ : 7.70-63.92) (Table 3).

\section{Multivariate analysis}

Considering Neo-Bioscore, the multivariate Cox regression analysis for tumor recurrence of non-pCR carcinoma showed that only the pathological stage was an independent prognostic factor. Taking the pathological

Table 2 Recurrence risk according to pathological response, Neo-Bioscore and Clustered Neo-Bioscore

\begin{tabular}{|c|c|c|c|c|c|c|}
\hline \multirow[t]{2}{*}{ Neo-Bioscore } & \multicolumn{2}{|l|}{ All patients } & \multicolumn{2}{|l|}{$\mathrm{pCR}$} & \multicolumn{2}{|l|}{ non-pCR } \\
\hline & $n=310$ & DFS-3y $(C 195 \%)^{a}$ & $n=43$ & DFS-3y (CI95\%) & $n=267$ & DFS-3y (CI95\%) \\
\hline 0 & $1(0.3 \%)$ & 1.00 & 0 & 1.00 & $1(0.4 \%)$ & 1.00 \\
\hline 1 & $18(5.8 \%)$ & 1.00 & $5(11.6 \%)$ & 1.00 & $13(4.9 \%)$ & 1.00 \\
\hline 2 & 47 (15.2\%) & $0.88(0.81-0.95)$ & 12 (27.9\%) & $0.89(0.79-0.99)$ & 35 (13.1\%) & $0.86(0.75-0.97)$ \\
\hline 3 & 84 (27.1\%) & $0.83(0.78-0.88)$ & $11(25.6 \%)$ & $0.91(0.82-1.00)$ & $73(27.3 \%)$ & $0.82(0.76-0.88)$ \\
\hline 4 & $83(26.8 \%)$ & $0.78(0.73-0.83)$ & $14(32.6 \%)$ & $0.93(0.87-1.00)$ & $69(25.8 \%)$ & $0.74(0.68-0.80)$ \\
\hline 5 & $49(15.8 \%)$ & $0.66(0.57-0.75)$ & $1(2.3 \%)$ & 1.00 & $48(18.0 \%)$ & $0.62(0.51-0.73)$ \\
\hline 6 & $23(7.4 \%)$ & $0.13(0.05-0.21)$ & 0 & - & $23(8.6 \%)$ & $0.13(0.05-0.21)$ \\
\hline 7 & $5(1.6 \%)$ & $0(\mathrm{mTFR}=475 \mathrm{~d})^{\mathrm{b}}$ & 0 & - & $5(1.9 \%)$ & $0(\mathrm{mTFR}=475 \mathrm{~d})$ \\
\hline Total & & $0.73(0.70-0.76)$ & Total & $0.92(0.88-0.96)$ & Total & $0.69(0.65-0.73)$ \\
\hline \multirow[t]{2}{*}{ Clustered Neo-Bioscore } & \multicolumn{2}{|l|}{ All patients } & \multicolumn{2}{|l|}{$\mathrm{pCR}$} & \multicolumn{2}{|l|}{ non-pCR } \\
\hline & $n=310$ & DFS-3y (CI95\%) & $n=43$ & DFS-3y (C195\%) & $n=267$ & DFS-3y (C195\%) \\
\hline $0-3$ & 150 & $0.87(0.83-0.91)$ & $28(65.1 \%)$ & $0.93(0.87-0.99)$ & $122(45.7 \%)$ & $0.86(0.82-0.90)$ \\
\hline $4-5$ & 132 & $0.73(0.68-0.78)$ & $15(34.9 \%)$ & $0.92(0.86-0.98)$ & $117(43.8 \%)$ & $0.70(0.65-0.75)$ \\
\hline 6 & $23(7.4 \%)$ & $0.13(0.05-0.21)$ & 0 & - & $23(8.6 \%)$ & $0.13(0.05-0.21)$ \\
\hline 7 & $5(1.6 \%)$ & $0(\mathrm{mTFR}=475 \mathrm{~d})$ & 0 & - & $5(1.9 \%)$ & $0(\mathrm{mTFR}=475 \mathrm{~d})$ \\
\hline
\end{tabular}

${ }^{\mathrm{a} D F S}$-3y 3-year disease-free survival rate ${ }^{\mathrm{b}}$ mTFR median time to the first recurrence ${ }^{c} p C R$ pathological complete response 

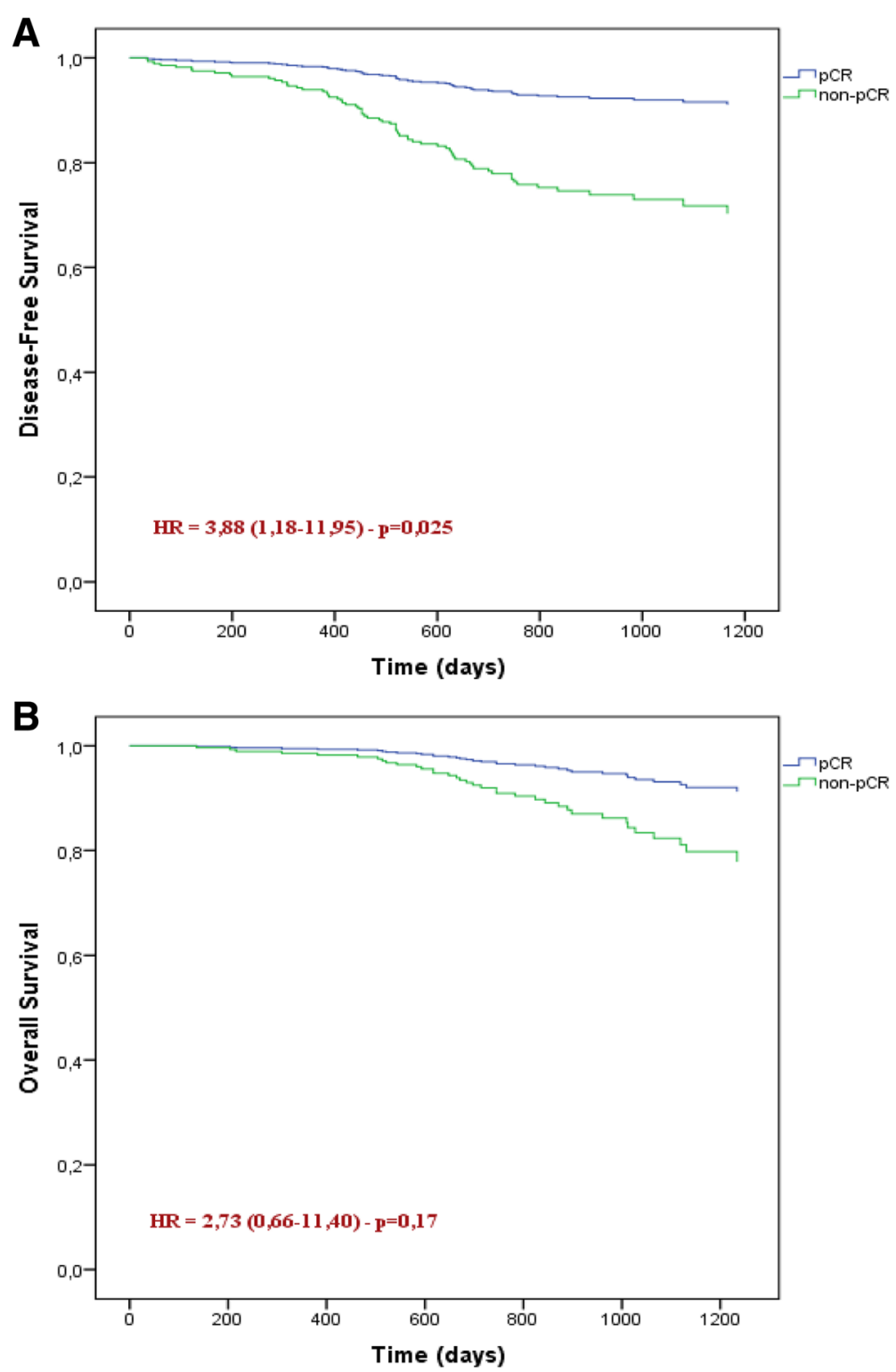

Fig. 1 Disease-Free Survival (a) and Overall Survival (b) according to pathological response

stage I as the reference, the pathological stage II had $\mathrm{HR}=8.12$ (CI95\%: 2.40-27.53) and the pathological stage III had $\mathrm{HR}=16.24$ (CI95\%: 4.71-55.95). Otherwise, the Clustered Neo-Bioscore presented distinct results with greater discrimination of independent pathological factors compared to Neo-Bioscore. For the low risk, the pathological stage III was the only independent prognostic factor $(\mathrm{HR}=13.0$; CI95\%: $1.6-$ 106.1). For the low-intermediate risk, TN subtype $(\mathrm{HR}=3.60 ; \quad \mathrm{CI95 \%}:$ 1.19-10.92) and pathological stages II (HR = 5.35; CI95\%: 1.19-24.01) and III (HR = 6.56; CI95\%: 1.29-33.32) were independent prognostic factors (Table 4 and Fig. 4).

\section{Discussion}

The Clustered Neo-Bioscore was a prognostic model of equivalent accuracy to Neo-Bioscore in women with non-pCR carcinoma but presented a more simplified categorization of scores, better differentiation among their prognostic scores and greater ability to identify independent prognostic factors.

Clustered Neo-Bioscore presented a prognostic accuracy in the risk of tumor recurrence with no statistically significant difference in relation to Neo-Bioscore for non-pCR breast carcinoma according to the ROC-time model. Otherwise, both models had greater prognostic accuracy compared than pathological stage $[19,20]$. 


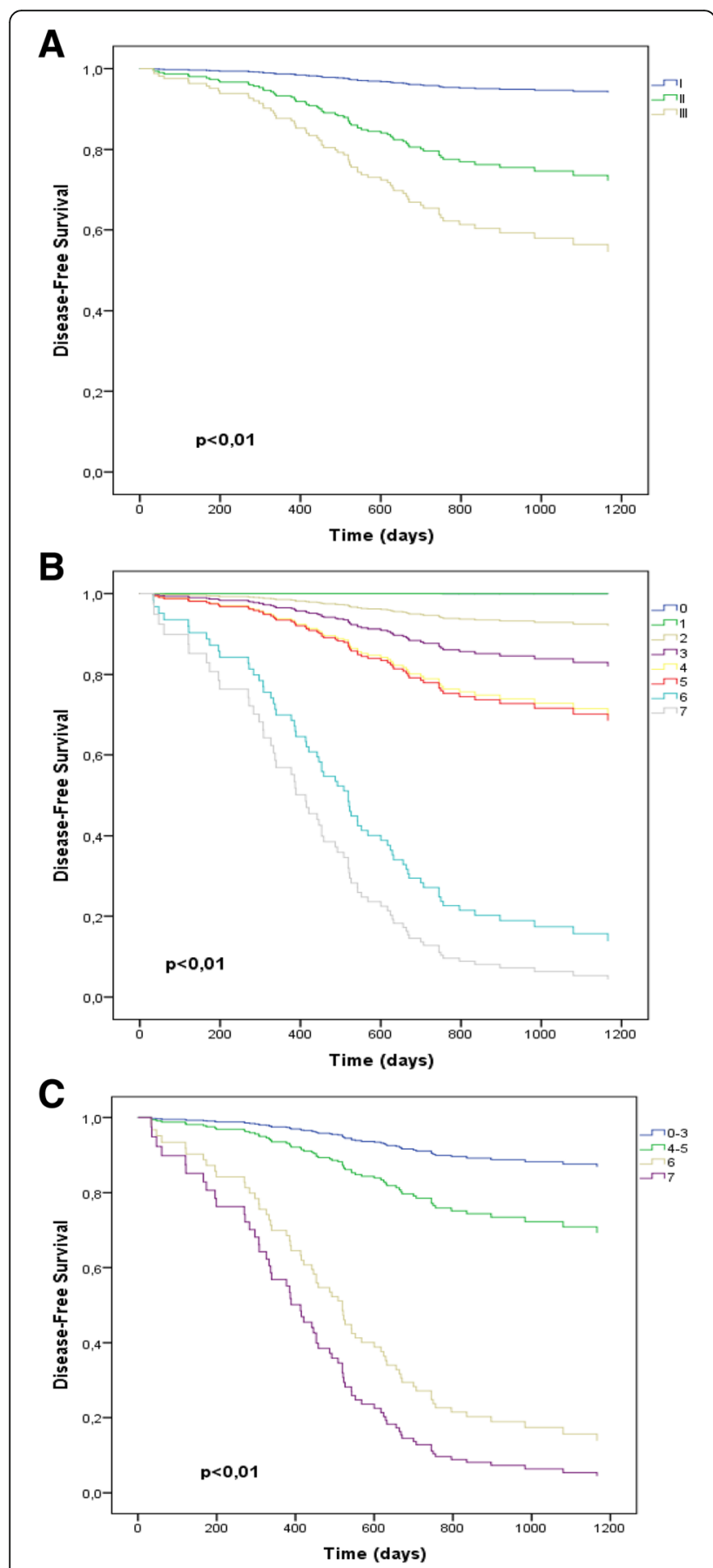

Fig. 2 Disease-Free Survival for non-pCR carcinoma according to pathological stage (a), Neo-Bioscore (b) and Clustered Neo-Bioscore (c)

Neo-Bioscore previously showed greater prognostic accuracy than pathological staging but this analysis did not exclusively involve non-pCR breast carcinoma [21, 22]. Therefore, the clustering of risk scores of Neo-Bioscore based on prognostic similarity did not bring about a loss of prognostic power to Clustered Neo-Bioscore. On the other hand, Clustered Neo-Bioscore has a simpler pattern with only four risk scores to allocate and define the prognosis of women undergoing NAC who have a worse prognosis because they have residual carcinoma in surgical specimen.

Most published clinical trials have evaluated the prognostic factors of women undergoing NAC with pCR carcinoma, therefore, a better oncological prognosis [25-27]. Otherwise, this trial turned to a better understanding of the prognostic factors for women with non-pCR carcinoma due to their worse survival. The main objective of this trial was to assess the prognosis of women with residual carcinoma after NAC. Previous data show that usually $80-90 \%$ of the women submitted to NAC present residual carcinoma in the surgical specimen. Unlike most of the trials that evaluated $\mathrm{pCR}$ carcinoma this trial focused on the prognostic factors of women who did not reach pCR [19-22].

Analysis of survival results by Mittendorf's pivotal trial [21] showed that the curves and survival at 3year for 10-year follow-up were very similar to the results obtained in our trial. About $13.8 \%$ of the women had non-pCR carcinoma that showed a 3.88 -fold greater risk of tumor recurrence than those with $\mathrm{pCR}$ carcinoma at 3-year follow-up $(p=0.025)$. However, there was no statistically significant difference for overall survival $(p=0.17)$ due to the shorter follow-up time since the survival curves between $\mathrm{PCR}$ and nonpCR carcinomas progressively move away from each other over time [23-26].

The subdivision of Clustered Neo-Bioscore into four risk scores was based on similar survival of NeoBioscore scores so that these new categories were independent and heterogeneous on prognosis. That is, the elevation of prognostic risk to tumor recurrence increased more linearly between the scores. Figure 2 showed that the survival curves for the risk scores of Clustered Neo-Bioscore were markedly distanced from each other, providing an adequate distinction of the prognostic value for tumor recurrence among the low, intermediate, high-intermediate, and high risk $(p<0.01)$ . According to Table 3, non-pCR breast carcinoma classified as high-intermediate or high risk by ClusteredNeo-Bioscore, although only $9 \%$ of the total, presented a risk of recurrence much higher than those classified as low or low-intermediate, which account for about $91 \%$ of the total. Taking the low risk as a reference, highintermediate and high risks had respectively 14-fold and 22 -fold greater risk of tumor recurrence. Otherwise, women classified as low-intermediate risk had about 2.6fold greater recurrence risk. Table 2 also showed that the distribution of risk scores in Clustered Neo-Bioscore at 3-years follow-up (DFS-3y) was more suitably heterogeneous to classify and distinguish women with non- 


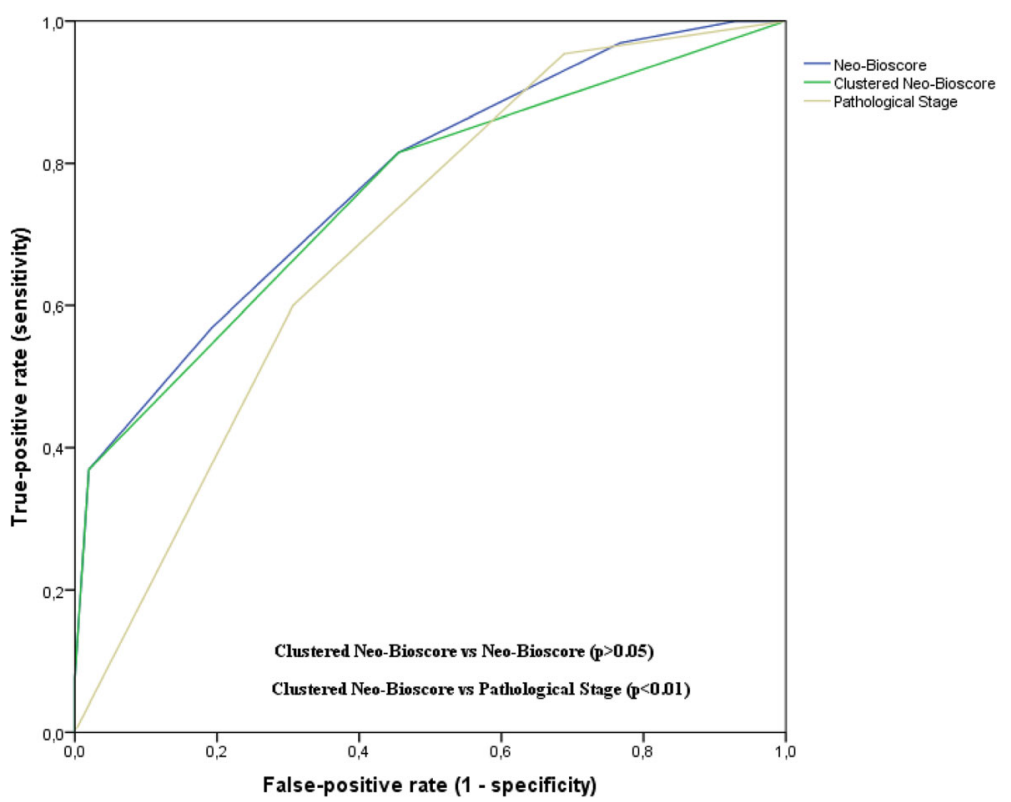

Fig. 3 Prognostic accuracy by time-ROC method for non-pCR carcinoma according to pathological stage, Neo-Bioscore and Clustered NeoBioscore. AUC - Area Under Curve. AUC Clustered Neo-Bioscore = 0.76 (C195\%: 0.69-0.83). AUC Neo-Bioscore = 0.78 (C195\%: 0.71-0.84). AUC Pathological stage $=0.69(\mathrm{Cl} 195 \%: 0.63-0.76)$

pCR carcinoma. The confidence intervals of the DFS-3y values for the risk scores in Clustered Neo-Bioscore do not overlap unlike the Neo-Bioscore scores of 0 to 5 .

Considering the Neo-Bioscore model, Cox regression showed that only the pathological stage was an independent prognostic factor for tumor recurrence in women with non-pCR breast carcinoma after NAC. The results showed that stages II and III were associated with 8-fold and 16-fold greater recurrence risk compared to the stage I. On the other hand, Neo-Bioscore did not identify any biological marker as a prognostic risk, contrary to what has been observed in some trials in which the biological nature of the tumor plays an important role in the prognosis [23, 25-28, 43]. Regarding Clustered Neo-Bioscore, Cox regression can only be performed for non-pCR carcinomas of low and lowintermediate risks which accounted for about $91 \%$ of total women. Non-pCR carcinomas of high-intermediate and high risks enrolled only 23 and 5 patients,

Table 3 Recurrence risk of non-pCR carcinoma according to Clustered Neo-Bioscore

\begin{tabular}{llll}
\hline Clustered Neo-Bioscore & $\mathrm{mTFR}^{\mathrm{a}}$ (days) & $p$ & $\mathrm{HR}^{\mathrm{b}}(\mathrm{Cl} 95 \%)$ \\
\hline Low & 1388 & Reference & \\
Low-intermediate & 1247 & $<0.01$ & $2.61(1.33-5.11)$ \\
High-intermediate & 618 & $<0.01$ & $14.05(6.78-29.11)$ \\
High & 433 & $<0.01$ & $22.19(7.70-63.92)$ \\
\hline
\end{tabular}

${ }^{a} m$ TFR median time to the first recurrence

${ }^{\mathrm{b}} \mathrm{HR}$ Hazard ratio at 3 years respectively, which made it impossible to perform the multivariate analysis.

Clustered Neo-Bioscore presented Cox regression results different from Neo-Bioscore with better power in discriminating independent prognostic factors. For nonpCR carcinoma of low risk, only pathologic stage III was an independent prognostic factor with 13-fold greater recurrence risk compared to the stage I. That is, only bulky tumor after NAC (greater than $5 \mathrm{~cm}$ and/or N2/N3) had worse prognosis in low-risk non-pCR carcinoma. Otherwise, non-pCR carcinoma of low-intermediate risk showed that TN subtype (3.60-fold) and pathological stages II (5.35-fold) and III (6.56-fold) had a greater risk of tumor recurrence. Some clinical trials have shown the prognostic risk of residual tumor after NAC but there is less information on the prognostic correlation between residual tumor volume and the biological factors of carcinoma as shown by the low-intermediate risk $[24,28,43]$. TN carcinoma is usually highly chemosensitivity and has high potential to reach pCR. However, results for non-pCR carcinoma of lowintermediate risk showed that $\mathrm{TN}$ subtype has a worse prognosis when it is associated with residual tumor after NAC (stages II and III) [16-22, 27, 43].

The results provided by Clustered Neo-Bioscore indicate the need to better identify prognostic factors associated with non-PCR breast cancer to evaluate new strategies related to NAC such as the number of chemotherapy cycles to be used, drug combination, assessment 
Table 4 Recurrence risk by Cox regression analysis of non-pCR carcinoma according to Neo-Bioscore and Clustered Neo-Bioscore of low and low-intermediate risks

\begin{tabular}{|c|c|c|c|}
\hline \multirow[t]{2}{*}{ Characteristics } & \multirow{2}{*}{$\begin{array}{l}\text { Neo-Bioscore } \\
\mathrm{HR}^{\mathrm{a}}(\mathrm{C} 195 \%) \\
\end{array}$} & \multirow{2}{*}{$\begin{array}{l}\text { Low risk } \\
\text { Clustered Neo-Bioscore } \\
\text { HR (C195\%) }\end{array}$} & \multirow{2}{*}{$\begin{array}{l}\text { Low-intermediate } \\
\text { Clustered Neo-Bioscore } \\
\text { HR (C195\%) }\end{array}$} \\
\hline & & & \\
\hline \multicolumn{4}{|l|}{ Menopausal status } \\
\hline Premenopausal & Reference & Reference & Reference \\
\hline Postmenopausal & $0.75(0.45-1.27)$ & $0.73(0.19-2.77)$ & $0.91(0.41-2.03)$ \\
\hline \multicolumn{4}{|l|}{ Histological grade } \\
\hline$|/| \mid$ & Reference & Reference & Reference \\
\hline III & $1.33(0.77-2.30)$ & $2.05(0.32-13.27)$ & $0.77(0.34-1.74)$ \\
\hline \multicolumn{4}{|l|}{ Ki-67 } \\
\hline $0-20 \%$ & Reference & Reference & Reference \\
\hline $20-50 \%$ & $2.29(0.68-7.73)$ & $0.76(0.12-4.79)$ & $2.39(0.29-19.89)$ \\
\hline$\geq 50 \%$ & 3.39 (0.99-11.58) & $4.81(0.41-56.41)$ & $3.37(0.41-27.57)$ \\
\hline \multicolumn{4}{|l|}{ Estrogen receptor } \\
\hline Allred 7-8 ( $\geq 70 \%)$ & Reference & Reference & Reference \\
\hline Allred 2-6 (<70\%) & $1.54(0.49-4.88)$ & $2.17(0.23-20.02)$ & $0.53(0.15-1.82)$ \\
\hline Negative & $1.35(0.44-4.15)$ & $1.33(0.11-16.67)$ & $1.39(0.16-12.50)$ \\
\hline \multicolumn{4}{|l|}{ HER2 } \\
\hline Negative & Reference & Reference & Reference \\
\hline Positive & $0.58(0.15-2.28)$ & $1.45(0.34-6.17)$ & $0.83(0.20-3.50)$ \\
\hline \multicolumn{4}{|l|}{ Tumor subtype } \\
\hline A & Reference & Reference & Reference \\
\hline$B$ & $0.50(0.12-2.12)$ & $0.53(0.09-3.20)$ & $1.10(0.49-2.50)$ \\
\hline HER2 & $0.58(0.15-2.28)$ & $1.45(0.34-6.17)$ & $0.83(0.20-3.50)$ \\
\hline $\mathrm{TN}$ & $1.90(0.49-7.30)$ & $8.92(0.94-84.93)$ & $3.60(1.19-10.92)$ \\
\hline \multicolumn{4}{|l|}{ Clinical stage } \\
\hline$|/| \mid$ & Reference & Reference & Reference \\
\hline III & $1.81(0.88-3.75)$ & $2.70(0.60-12.21)$ & $1.00(0.38-2.66)$ \\
\hline \multicolumn{4}{|l|}{ Pathological stage } \\
\hline I & Reference & Reference & Reference \\
\hline$\|$ & $8.12(2.40-27.53)$ & $4.17(0.47-37.33)$ & $5.35(1.19-24.01)$ \\
\hline III & $16.24(4.71-55.95)$ & $13.00(1.60-106.10)$ & $6.56(1.29-33.32)$ \\
\hline
\end{tabular}

${ }^{\mathrm{a}} H R$ Hazard ratio at 3 years

of tumor downstaging, identification of new biomarkers of tumor resistance or new protocols to complement the NAC in order to reduce the risk of tumor recurrence. The breast carcinomas analyzed in this clinical trial presented high-risk clinical and biological characteristics predominating size $\mathrm{T} 3 / \mathrm{T} 4$ size, positive axilla, clinical stage III, histological grade III, non-luminal carcinoma A, negative ER and Ki-67 $\geq 20 \%$.

\section{Conclusions}

In summary, Clustered Neo-Bioscore demonstrated potential as a better marker for prognostic analysis of non-
pCR carcinoma undergoing NAC, since it had a prognostic accuracy equivalent to Neo-Bioscore, but presented a more simplified format in the subdivision of risk scores, a better model to discriminate the prognosis among the risk scores due to the greater prognostic differentiation and a better pattern in the assessment of independent prognostic factors in women classified as low and low-intermediate risks.

This is a retrospective clinical trial with limitations inherent to bias control and confounding factors, but we believe that it makes an important scientific contribution to understanding the prognostic factors of 

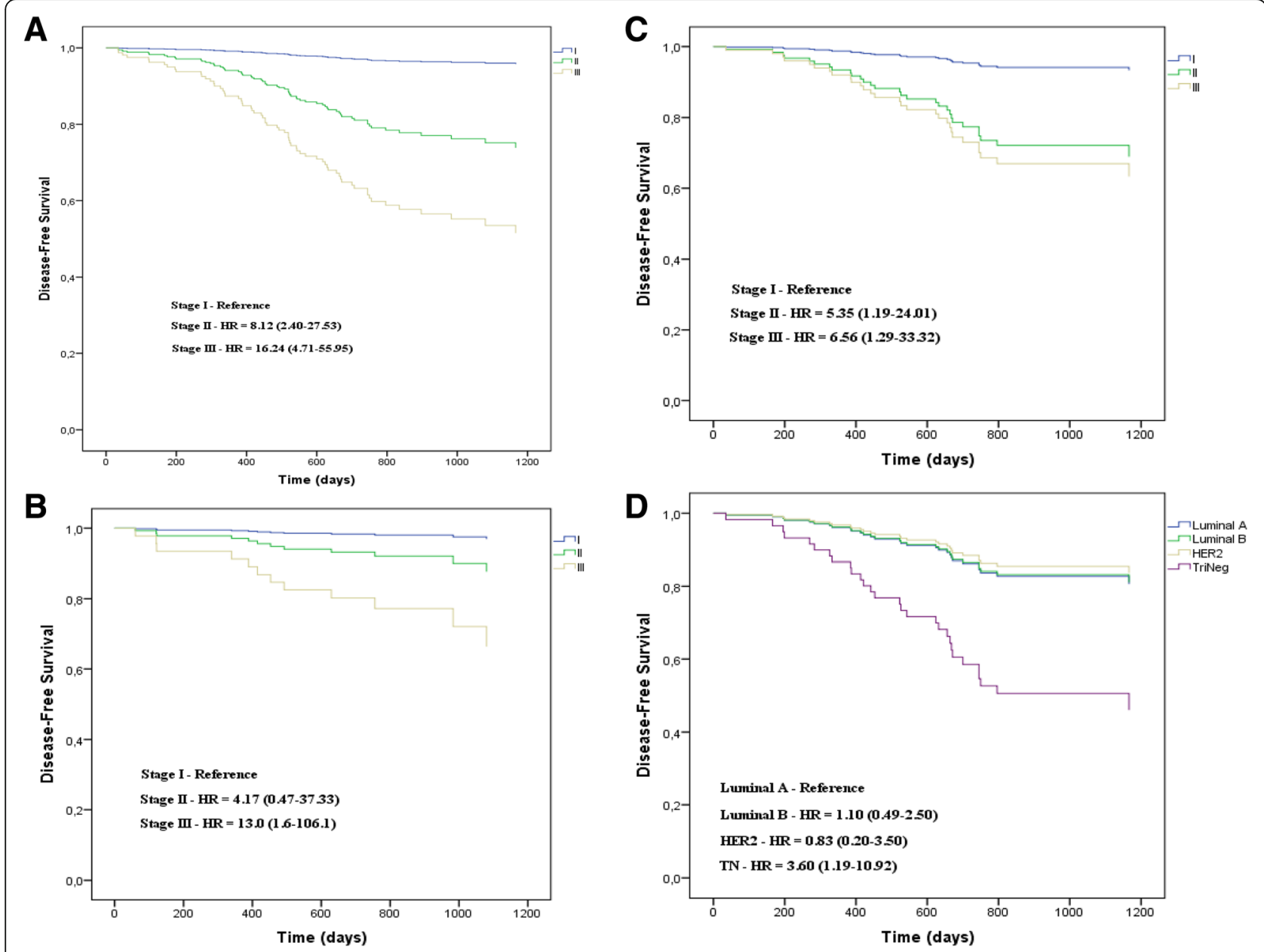

Fig. 4 Independent prognostic factors of non-pCR carcinoma according to Neo-Bioscore (a), low (b) and low-intermediate Clustered Neo-Bioscore (c, d)

non-pCR breast carcinoma. External validation trials should be performed to evaluate the generality of the results obtained.

\section{Additional file}

Additional file 1: Datasets supporting the study findings. (XLSX $122 \mathrm{~kb}$ )

\section{Abbreviations}

AC: Doxorubicin plus cyclophosphamide; AUC: Area under the curve; CAISM: Woman's Hospital Dr. José Aristodemo Pinotti; DFS: Disease-free survival; ER: Estrogen receptor; FCM: Faculty of Medical Sciences; HER2: Human epidermal growth factor; HR: Hazard ratio; Ki-67: Cellular proliferation index; mOS: Median overall survival; mTFR: Median time from first recurrence; NAC: Neoadjuvant chemotherapy; non-pCR: Pathological non-complete response; OS: Overall survival; pCR: Pathological complete response; PR: Progesterone receptor; ROC: Receiver operating characteristic; T: Paclitaxel; TN: Triple-negative; UNICAMP: State University of Campinas

\section{Acknowledgements}

Not applicable.

\section{Authors' contributions}

UR, CC, SOBR and LCZ have made substantial contributions to the study design and to the analysis and interpretation of the data. They have critically reviewed the manuscript and given approval to the submitted and final versions. They have also contributed to the inclusion of patients and acquisition of data.

\section{Funding}

This work was not supported by official or private organizations. The authors would like to acknowledge UNICAMP for supporting the study.

Availability of data and materials

The dataset supporting the conclusions of this article is included as an Additional file 1.

\section{Ethics approval and consent to participate}

This study was approved by the Ethics and Research Committee of Unicamp (No. 2.238.253). For this type of study (retrospective data analysis), the application of formal consent was disclaimed by the Research Ethics Committee. All procedures in the current study were in accordance with the ethical standards of the Institutional Research Committee and with the 1964 Helsinki Declaration and its later amendments.

\section{Consent for publication}

Not applicable.

\section{Competing interests}

The authors declare that they have no competing interests. 
Received: 30 January 2018 Accepted: 10 June 2019

Published online: 17 June 2019

\section{References}

1. DeSantis CE, et al. Cancer treatment and survivorship statistics. CA Cancer J Clin. 2016;66(4):271-89. https://doi.org/10.3322/ccac.21349.

2. Nishimura $R$, et al. Evaluation of factors related to late recurrence - later than 10 years after the inicial treatment - in primary breast cancer. Oncology. 2013;85:100-10. https://doi.org/10.1159/000353099.

3. Thompson AM, et al. Neoadjuvant treatment of breast cancer. Ann Oncol. 2012;23(10):x231-6. https://doi.org/10.1093/annonc/mds324.

4. Roughton $\mathrm{MC}$, et al. Optimizing delivery of breast conservation therapy: a multidisciplinary approach to oncoplastic surgery. Ann Plast Surg. 2012; 69(3):250. https://doi.org/10.1097/SAP.0b013e31822afa99.

5. Ribeiro J, et al. Optimal approach in early breast cancer: adjuvant and neoadjuvant treatment. EJC Suppl. 2013;11(2):3-22. https://doi.org/10.1016/j. ejcsup.2013.07.029

6. Bardia A, Baselga J. Neoadjuvant therapy as a platform for drug development and approval in breast cancer. Clin Cancer Res. 2013;19(23): 6360-70. https://doi.org/10.1158/1078-0432.CCR-13-0916.

7. Faneyte IF, et al. Breast cancer response to neoadjuvant chemotherapy: predictive markers and relation with outcome. Br J Cancer. 2003;88:406-12. https://doi.org/10.1038/sj.bjc.6600749.

8. The Cancer Genome Atlas Network. Comprehensive molecular portraits of human breast tumours. Nature. 2012;490(7418):61-70. https://doi.org/10. 1038/nature11412

9. Pusztai $L$, et al. Molecular classification of breast cancer: limitations and potential. Oncologist. 2006;11(8):868-77. https://doi.org/10.1634/ theoncologist.11-8-868.

10. Sorlie T. Introducing molecular subtyping breast cancer into the clinic? J Clin Oncol. 2009;27(8):1153-4. https://doi.org/10.1200/JCO.2008.20.6276.

11. Von Minckwitz G, et al. Definition and impact of pathologic complete response on prognosis after neoadjuvant chemotherapy in various intrinsic breast cancer subtypes. J Clin Oncol. 2012;30(15):1796-804. https://doi.org/ 10.1200/JCO.2011.38.8595.

12. Ring, et al. Oestrogen receptor status, pathological complete response and prognosis in patients receiving neoadjuvant chemotherapy for early breast cancer. Br J Cancer. 2004;91(12):2012-7. https://doi.org/10.1038/sj.bjc. 6602235.

13. Jones $\mathrm{RL}$, et al. Relationship between oestrogen receptor status and proliferation in predicting response and long-term outcome to neoadjuvant chemotherapy for breast cancer. Breast Cancer Res Treat. 2010;119:315-29. https://doi.org/10.1007/s10549-009-0329-x.

14. van de Ven $S$, et al. Discordances in ER, PR and HER2 receptors after neoadjuvant chemotherapy in breast cancer. Cancer Treat Rev. 2011;37(6): 422-30. https://doi.org/10.1016/j.ctrv.2010.11.006.

15. Cheang MCU, et al. Ki67 index, HER2 status, and prognosis of patients with luminal B breast cancer. J Natl Cancer Inst. 2009;101(10):736-50. https://doi. org/10.1093/jnci/djp082.

16. Yerushalmi $\mathrm{R}$, et al. Ki67 in breast cancer: prognostic and predictive potencial. Lancet Oncol. 2010;11:174-83. https://doi.org/10.1016/S14702045(09)70262-1.

17. Goldhirsch A, et al. Personalizing the treatment of women with early breast cancer: highlights of the St Gallen international expert consensus on the primary therapy of early breast Cancer. Ann Oncol. 2013;24(9):2206-23. https://doi.org/10.1200/JCO.2011.38.8595.

18. Untch M, et al. 13th St. Gallen international breast Cancer conference 2013: primary therapy of early breast cancer evidence, controversies, consensus opinion of a german team of experts. Breast Care (Basel). 2013;8(3):221-9. https://doi.org/10.1159/000351692.

19. Jeruss JS, et al. Combined use of clinical and pathologic staging variables to define outcomes for breast cancer patients treated with neoadjuvant therapy. J Clin Oncol. 2008;26(2):246-52. https://doi.org/10.1200/JCO.2007. 11.5352.

20. Marmé $F$, et al. Utility of the CPS+EG staging system in hormone receptorpositive, human epidermal growth factor receptor 2-negative breast cancer treated with neoadjuvant chemotherapy. Eur J Cancer. 2016;53:65-74. https://doi.org/10.1016/j.ejca.2015.09.022.

21. Mittendorf EA, et al. The neo-bioscore update for staging breast cancer treated with neoadjuvant therapy. Incorporation of prognostic biologic factors into staging after treatment. JAMA Oncol. 2016;2(7):929-36. https:// doi.org/10.1001/jamaoncol.2015.6478.

22. Mittendorf EA, et al. Validation of a novel staging system for diseasespecific survival in patients with breast cancer treated with neoadjuvant therapy. J Clin Oncol. 2016;29(15):1956-62. https://doi.org/10.1200/JCO. 2010.31.8469.

23. Earl H, et al. Neoadjuvant trials in early breast cancer: pathological response at surgery and correlation to longer term outcomes - what does it all mean? BMC Med. 2015;22:234. https://doi.org/10.1186/s12916015-0472-7.

24. Symmans WF, et al. Measurement of residual breast cancer burden to predict survival after neoadjuvant chemotherapy. J Clin Oncol. 2007;25: 4414-22. https://doi.org/10.1200/JCO.2007.10.6823.

25. Gajdos C, et al. Relationship of clinical and pathologic response to neoadjuvante chemotherapy and outcome of locally advanced breast cancer. J Surg Oncol. 2002;80:4-11. https://doi.org/10.1002/jso.10090.

26. Pennisi $A$, et al. Relevance of pathologic complete response after neoadjuvant therapy for breast cancer. Breast Cancer (Auckl). 2016;10:103-6. https://doi.org/10.4137/BCBCR.S33163.eCollection.

27. Spring L, et al. Abstract 1439: Pathological complete response after neoadjuvant chemotherapy predicts improved survival in all major subtypes of breast cancer: systematic review and meta-analyses of over 18,000 patients. AACR 107th Annual Meeting 2016; April 16-20; 2016. https://doi. org/10.1158/1538-7445.AM2016.

28. Sheri $A$, et al. Residual proliferative cancer burden to predict long-term outcome following neoadjuvant chemotherapy. Ann Oncol. 2015;26(1):7580. https://doi.org/10.1093/annonc/mdu508

29. Brenton JD, et al. Molecular classification and molecular forecasting of breast cancer: ready for clinical application? J Clin Oncol. 2005;23(29):735060. https://doi.org/10.1200/JCO.2005.03.3845.

30. Dekker TJA, et al. Reliability of core needle biopy for determining ER and HER2 status in breast cancer. Ann Oncol. 2013;24:931-7. https://doi.org/10. 1093/annonc/mds599.

31. Woff AC, et al. Recommendations for human epidermal growth fator receptor 2 testing in breast cancer: American Society of Clinical Oncology/College of American Pathologists clinical practice guideline update. J Clin Oncol. 2013;31(31):3997-4013. https://doi.org/10.1200/JCO. 2013.50.9984.

32. Hammond ME, et al. American Society of Clinical Oncology/college of American pathologists guideline recommendations for immunohistochemical testing of estrogen and progesterone receptors in breast cancer. J Clin Oncol. 2010;28(16):2784-95. https://doi.org/10.1200/ ICO.2009.25.6529

33. Luporsi $\mathrm{E}$, et al. Ki-67: level of evidence and methodological considerations for its role in the clinical management of breast cancer: analytical and critical review. Breast Cancer Res Treat. 2012;132(3):895-915. https://doi.org/ 10.1007/s10549-011-1837-z.

34. Kim Kl, et al. Ki-67 as a predictor of response to neoadjuvant chemotherapy in breast cancer patients. J Breast Cancer. 2014;17(1):40-6. https://doi.org/10 4048/jbc.2014.17.1.40.

35. Sparano JA, et al. Weekly paclitaxel in adjuvant treatment of breast cancer. N Engl J Med. 2008;358(16):1663-71. https://doi.org/10.1056/ NEJMoa0707056.

36. Romond EH, et al. Trastuzumab plus adjuvant chemotherapy for operable HER2 positive breast cancer. N Engl J Med. 2005;353:1673-84. https://doi. org/10.1056/NEJMoa052122.

37. Gogas $\mathrm{H}$, et al. Paclitaxel and carboplatin as neoadjuvant chemotherapy in patients with locally advanced breast cancer: a phase II trial of the Hellenic cooperative oncology group. Clin Breast Cancer. 2010;10(3):230-7. https:// doi.org/10.3816/CBC.2010.n.031.

38. Edge SB, et al. The American joint committee on Cancer: the 7ht edition of the AJCC cancer staging manual and the future of TNM. Ann Surg Oncol. 2010;17:1471-4. https://doi.org/10.1245/s10434-010-0985-4.

39. Van Merten ELP, et al. Recist revised: implications for the radiologist. A review article on the modified RECIST guideline. Eur Radiol. 2010;20(6):145667. https://doi.org/10.1007/s00330-009-1685-y.

40. Freedman DA. Statistical models: theory and practice. New York: Cambridge University Press: 2012

41. Sen PK, et al. Review: contemporary textbooks on multivariate statistical analysis: a panoramic appraisal and critique. J Am Stat Assoc. 1989:81(394): $560-4$. 
42. Blanche $P$, et al. Estimating and comparing time-dependent areas under receiver operating characteristic curves for censored event times with competing risks. Stat Med. 2013;32(30):5381-97. https://doi.org/10.1002/sim. 5958.

43. Ear $\mathrm{H}$, et al. Neoadjuvant trials in early breast cancer: pathological response at surgery and correlation to longer term outcomes - what does it all mean? BMC Med. 2015;13:234. https://doi.org/10.1186/s12916-015-0472-7.

\section{Publisher's Note}

Springer Nature remains neutral with regard to jurisdictional claims in published maps and institutional affiliations.

Ready to submit your research? Choose BMC and benefit from:

- fast, convenient online submission

- thorough peer review by experienced researchers in your field

- rapid publication on acceptance

- support for research data, including large and complex data types

- gold Open Access which fosters wider collaboration and increased citations

- maximum visibility for your research: over $100 \mathrm{M}$ website views per year

At BMC, research is always in progress.

Learn more biomedcentral.com/submissions 